\title{
Ileoileal intussusception as initial manifestation of Crohn's disease
}

\author{
López-Tomassetti Fernández E. M.*†, Lorenzo Rocha N.†, Arteaga González I.†, \\ Carrillo Pallarés A ${ }^{\dagger}$
}

\begin{abstract}
Intussusception is usually considered a childhood condition, but it may also be present in adults, where it is more often associated with an underlying pathology. There is no agreement upon the correct treatment of adult intussusception, although surgical intervention is considered necessary. Resection without prior reduction has been the traditional treatment of choice due to the significant risk for malignancy found in most series. We describe an unusual case of intestinal necrosis secondary to ileoileal intussusception caused by Crohn's disease. A long intestinal resection was necessary and the patient was discharged without major complications. Based on the details of this case, the authors emphasize the potential importance of considering individualized treatment of adult intussusception. The practical benefit for reduction of viable bowel in Crohn's patients is the preservation of bowel length.
\end{abstract}

Key words: Bowel obstruction, Crohn's disease, Intussusception, Acute abdomen.

\section{INTRODUCTION}

Crohn's disease is a chronic granulomatous inflammatory disease that may involve any part of the alimentary tract, although the ileum is the most frequent location. The presenting symptoms are variable and include abdominal pain, growth failure, and, less commonly, diarrhea. In the early stages, Crohn's disease causes small erosions on the inner surface of the bowel (aphthous ulcers). With time, the erosions become deeper and larger, causing scarring and stiffness of the bowel. Histologically, the active mucosal lesion shows a mixed infiltrate of lymphocytes, plasma cells, and neutrophils. Non-necrotizing granulomas are found in

* To whom correspondence should be addressed: Dr. Eudaldo LópezTomassetti Fernández. Medical doctor. Department of surgery, Hospital Universitario de Canarias. Ofra, s/n. La Cuesta, $38320 \mathrm{La}$ Laguna. Santa Cruz de Tenerife. Telephon. +34922678700

E-mail: drtomassetti@wanadoo.es

$\dagger$ Department of Gastrointestinal Surgery. Hospital Universitario de Canarias (HUC), Canary Islands, Spain about $50 \%$ of resection specimens and may be located in any portion of the bowel wall. Crohn's disease was shown to be a predisposing factor for ileoileal intussusception (1).

Intussusception occurs when a proximal segment of bowel telescopes into an adjacent distal segment. Unlike in children, it is an uncommon cause of small bowel obstruction in adults. Causes include any lesion within the intestinal lumen or wall which alters peristalsis.

We describe an unusual case of acute intestinal obstruction due to ileoileal intussusception as an initial manifestation of Crohn's disease. We speculate that intussusception was secondary to inflammatory edema and spasm, which led to impaired contraction of the bowel and allowed unbalanced peristaltic forces to rotate the intestinal wall inwards and initiate the invagination. Hence, Crohn's disease should be taken into consideration in young patients with intestinal intussusception if no other cause is found. 


\section{CASE HISTORY}

A 20-year-old patient presented to emergency with complaints of central abdominal pain, vomitting, and bloody stool for the previous 2 days. The patient was afebrile and his vital signs were stable. He was dehydrated, with no external signs of anaemia and appeared well. Rectal examination was unremarkable. The abdomen was distended and examination revealed mild central abdominal tenderness with diminished bowel sounds. He had a BMI of 21 and no past medical history. Routine investigations showed a raised white cell count of $21,200 / \mathrm{mm}^{3}$, and a mild metabolic acidosis ( $\mathrm{pH}$ of 7.34, serum level of bicarbonate of $18.12 \mathrm{mmol} / \mathrm{L}$ ). Hematocrit was $41 \%$ and haemoglobin was $13.6 \mathrm{~g} / \mathrm{dL}$. An abdominal X-ray showed grossly distended loops of small intestine with absent colonic shadows, suggestive of mid-bowel obstruction (Figure $1)$.

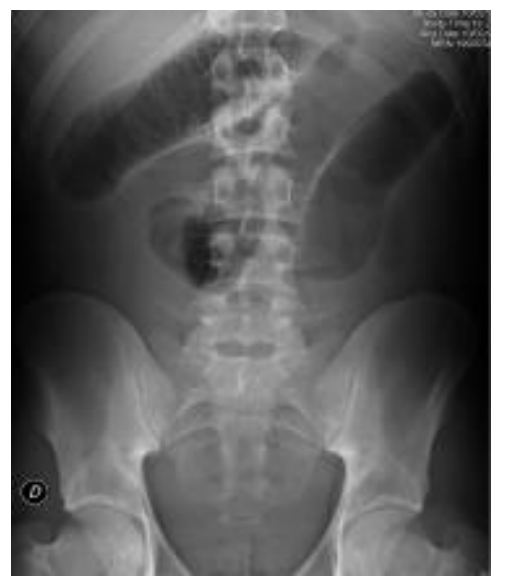

Figure 1. Abdominal X-ray revealed distended bowel loops, with airfluid levels.

Conservative treatment with nasogastric tube and endovenous fluids was initially instaured for at least 36 hours but ultrasound was later considered because there was no improvement.

Ultrasonography showed a moderate amount of free fluid in the peritoneal cavity with distended bowel loops and enlarged lymph nodes in the mesentery. A CT-scan was recommended by the radiologist. Contrast enhanced CT-scan (Figure 2) revealed a hypodense mass lesion in the pelvis, representing a thickened segment of bowel (enteroenteric intussusception).

The decision to recommend operative intervention to the patient was made and finally accepted. Laparotomy revealed a moderate amount of serous free fluid, grossly distended proximal small bowel loops, and distal ileum intussusception. Enlarged lymph nodes were noted at the site of the intussusception. The intussusceptum segment (60 cm-long ileum) was reduced. It showed transmural ischemic necrosis. Finally, a $70 \mathrm{~cm}$-long resection with side to side isoperistaltic anastomosis was carried out. Postoperative period was uneventful for the patient and he was discharged on the fifth day after surgery.

Gross examination of the specimen showed extensive (60 cm-long) bowel infarction. The rest of the bowel (10 cm-long) was hemorrhagic and presented small longitudinal ulcerations with irregular borders in the mucosa (Figure 3).

Microscopy revealed extensive infarcted hemorrhage. Peripherally, a $10 \mathrm{~cm}$-long bowel without necrosis was further studied. It showed pathological changes similar to Crohn's disease. The mucosa was infiltrated with neutrophils. Transmural inflammation, deep ulcerations, and non-necrotizing epithelioid cell granuloma (sarcoid like-granulomas) were demonstrated (Figure 4). Resected lymph nodes displayed granulomas with fibrotic changes. A final diagnosis of inflammatory Crohn's disease affecting the ileum was made.

At 6 months follow up, the patient was doing well and was receiving mesalazine $2 \mathrm{~g}$ orally for the treatment of Crohn's disease.
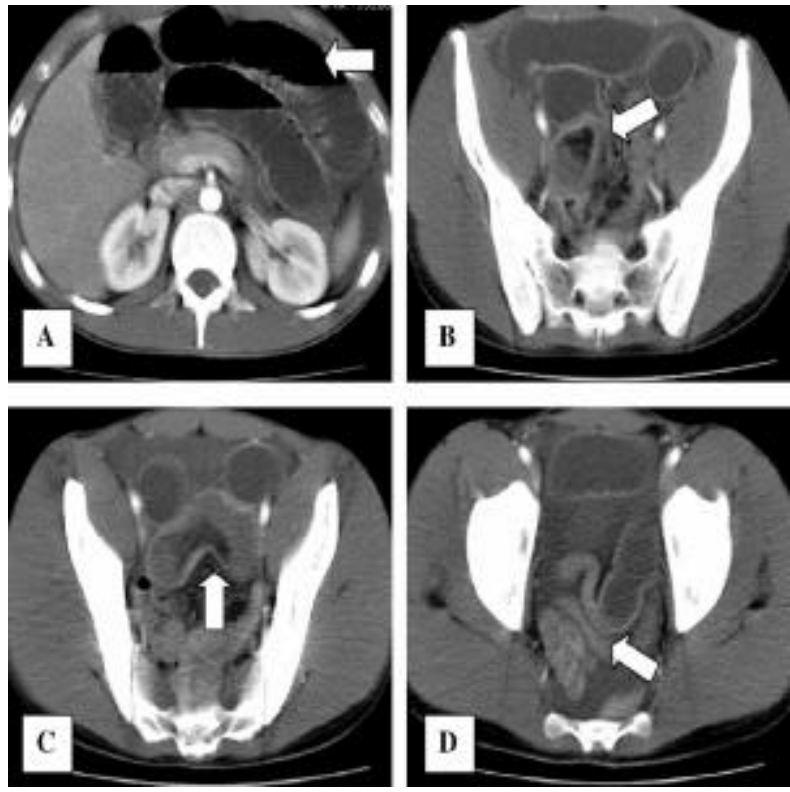

Figure 2. Contrast CT-scan showed:

A. Distended proximal bowel loops, with air-fluid levels.

B. Fat pulled into the intussusception (white arrow) adjacent to a portion of the intussusceptum.

C. Enteroenteric intussusception forming a loop within the pelvis. Intussuscipiens containing intussusceptum in longitudinal section.

D. Distal bowel loops were not dilated. Moderate amount of free fluid.

\section{DISCUSSION}

Crohn's disease has been linked to a variety of 

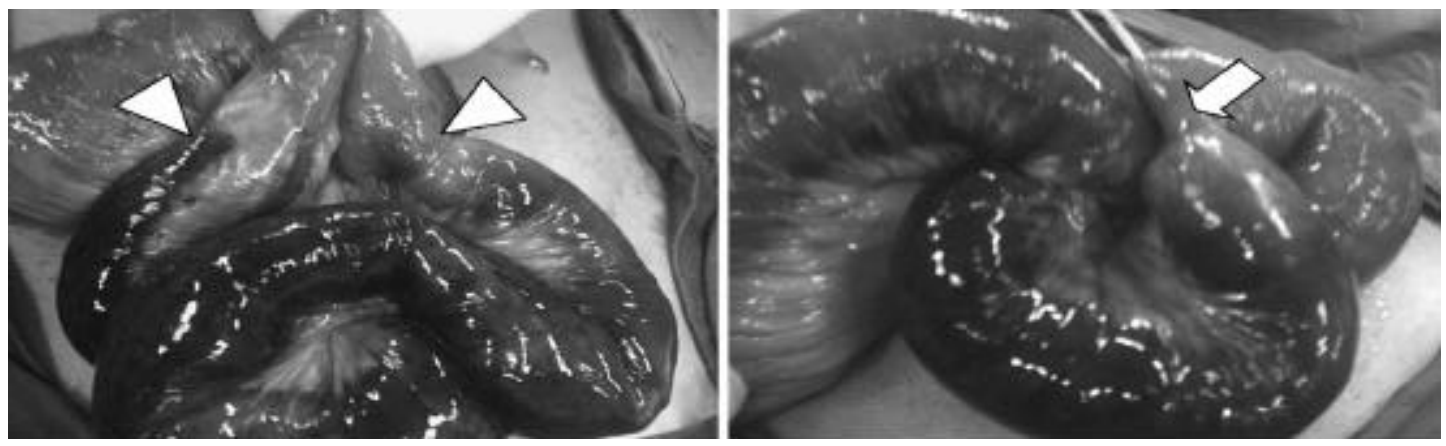

Figure 3. Laparotomy revealed:

Ileoileal intussusception. Initial point of intussusception is showed with arrow (affected by Crohn's disease). Intussusceptum bowel was necrotic with thin walls and close to perforate.
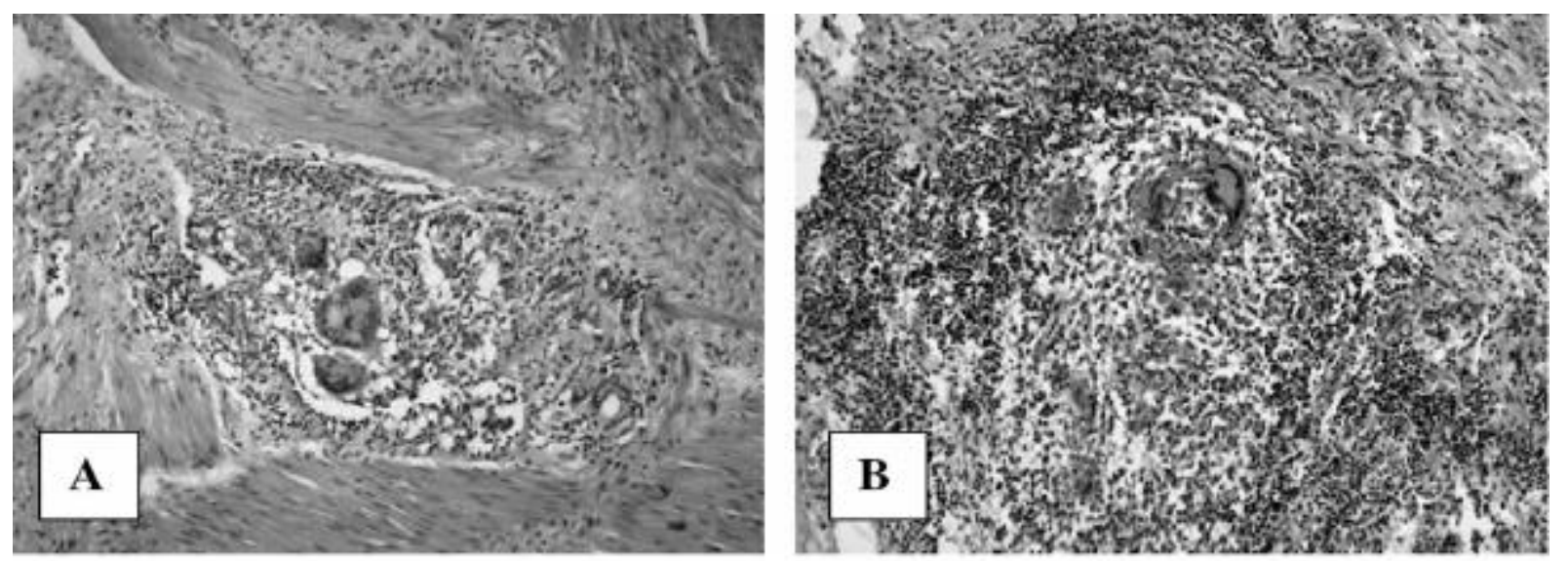

Figure 4. Microscopy examination of the specimen (H\&E, 100x):

A. Thickening of muscularis mucosae with granulomas occurs near the obstructive point.

B. Granulomas were present in the mucosa.

presentations and complications. One of the most common complications leading to surgery is chronic intestinal obstruction secondary to chronic inflammation with fibrous scar, which is rarely complete but is high grade (2). Cases of intestinal obstruction secondary to small bowel intussusception by a segment of active Crohn's ileitis have been rarely reported $(1,3)$.

Intussusception is an uncommon cause of intestinal obstruction in adults. It occurs when a proximal segment of bowel telescopes into an adjacent distal segment. Symptoms are usually the same as for other types of intestinal obstruction. The classic triad of abdominal mass, pain, tenderness, and bloody stool is rarely found. The aetiology of intussusception in the small bowel and the colon are different.

In two thirds of cases, intussusception arises in the small bowel and is more common in the proximal bowel due to increased peristaltic activity.

About $10 \%$ of intussusceptions in adults are primary and $80-90 \%$ are secondary to an underlying pathology.
Of the latter, approximately $65 \%$ are secondary to a benign lesion or malignant tumor, and $15-20 \%$ of cases are due to processes other than tumor growth.

With the widespread use of CT, the diagnosis is nowadays most often made by the radiologist since the CT features of intussusception are virtually pathognomonic $(5,6)$.

Optimal management between reduction and primary resection in treatment of adult intussusception has caused some controversy. Some authors have established the principle of resection without reduction whenever possible based on a high incidence of underlying malignancy that could not be confirmed either preoperatively or intraoperatively (7). The problems against reduction include: risk of intraluminal seeding or venous embolization, possible perforation during manipulation, and subjection of the patient to increased risk of anastomotic leakage due to edematous bowel. In favor of reduction is the preservation of a theoretically viable bowel.

Nowadays, it is recommended to use a selective 
approach to resection that takes into consideration the site of intussusception that tends to influence the type of pathology (8).

We advocate individualized treatment, depending on medical history and findings during intervention, as some cases of transient and acute intussusception have been observed on small bowel barium studies in patients with Crohn's disease, adult coeliac disease, and many other benign conditions.

Transient intussusception has been observed occasionally on small bowel barium studies in patients with Crohn's and adult coeliac disease. The leading point to the intussusception in Crohn's Disease is probably the dysrhythmic contractions secondary to the on-going inflammatory process of a thickened, inflamed segment of bowel wall (9). Knowles described transient intussusception in two patients with Crohn's disease. In both cases, a repeat CT showed resolution of the intussusception. Both patients underwent exploratory laparotomy, and no intraluminal mass was detected (10).

Crohn's disease remains a common cause of short bowel syndrome. The risk of developing short bowel syndrome in patients with Crohn's disease is approximately $5 \%$ to $10 \%$ in reported series. As far as we know, this is the first case reported of small bowel intussusception as an initial manifestation of Crohn's disease, for which long surgical resection was necessary and thus worsened the prognosis of the disease. The incidence of short bowel syndrome (SBS) varies depending on the underlying disease process and its medical and surgical management. Aggressive resectional therapy, surgical complications, and errors in initial diagnosis contribute to development of SBS in these patients (11).

\section{CONCLUSION}

We conclude that small bowel intussusception might be the first manifestation of Crohn's disease. Intussusception might be secondary to impaired contraction of the bowel associated with inflammation, edema and spasm. Management of small bowel intussusception in young patients should be individualized depending on the patient's medical history and intraoperative findings. In these cases, early exploration and reduction of the intussusception (thus avoiding intestinal necrosis) may prevent the onset of short bowel syndrome in the future.

We recommend urgent CT-scan in patients with a known history of Crohn's disease and a sudden onset of abdominal pain to rule out intussusception and its potential complications. In these cases, abdominal ultrasound might not be able to detect these problems.

\section{ACKNOWLEDGEMENT}

We acknowledge the help of Alvaro Blanch in preparing the English language version of the manuscript.

\section{REFERENCES}

1. Shah A, Roberts J, Lipsky H, et al. Enteroenteric intussusception: an unusual presentation of Crohn's disease in an adult patient. Am J Gastroenterol 1995; 90:2231-2.

2. Wolff B. Surgery for distal small bowel, colonic, and anorectal crohn's disease. In: Zuidema GD, Yeo CJ (editors): Shackelford's Surgery of the Alimentary Tract. Philadelphia: WB Saunders, 2002, pp 105-116.

3. Cohen DM, Conard FU, Treem WR, et al. Jejunojejunal intussusception in Crohn's disease. J Pediatr Gastroenterol Nutr 1992;14:101-3.

4. Begos DG, Sandor A, Modlin IM. The diagnosis and management of adult intussusception. Am J Surg 1997;173:8894.

5. Huang BY, Warshauer DM. Adult intussusception: diagnosis and clinical relevance. Radiol Clin North Am 2003;41:1137-51.

6. Byrne AT, Geoghegan T, Govender P, et al. The imaging of intussusception. Clin Radiol 2005;60:39-46.

7. Weilbaecher D, Bolin JA, Hearn D, et al. Intussusception in adults. Review of 160 cases. Am J Surg 1971;121:531-5.

8. Eisen LK, Cunningham JD, Aufses AH Jr. Intussusception in adults: institutional review. J Am Coll Surg 1999;188:390-5.

9. Kihiczak D, Rosenfeld DL. Crohn's disease presenting as intermittent ileocolic intussusception. Clin Pediatr 1998;37:6358.

10. Knowles MC, Fishman EK, Kuhlman JE, et al. Transient intussusception in Crohn's disease: CT evaluation. Radiology 1989; 170:814.

11. Yamamoto T, Allan RN, Keighley MR. Long-term outcome of surgical management for diffuse jejunoileal Crohn's disease. Surgery 2001;129:96-102.

Eudaldo M. López-Tomassetti Fernándezis a third year surgical resident at University Hospital of The Canary Islands in Spain. He has special interest in Gastrointestinal Surgical Oncology and Bariatric Surgery. 


\section{The McGill Journal of \\ Medicine is proud to announce a new collaboration with}

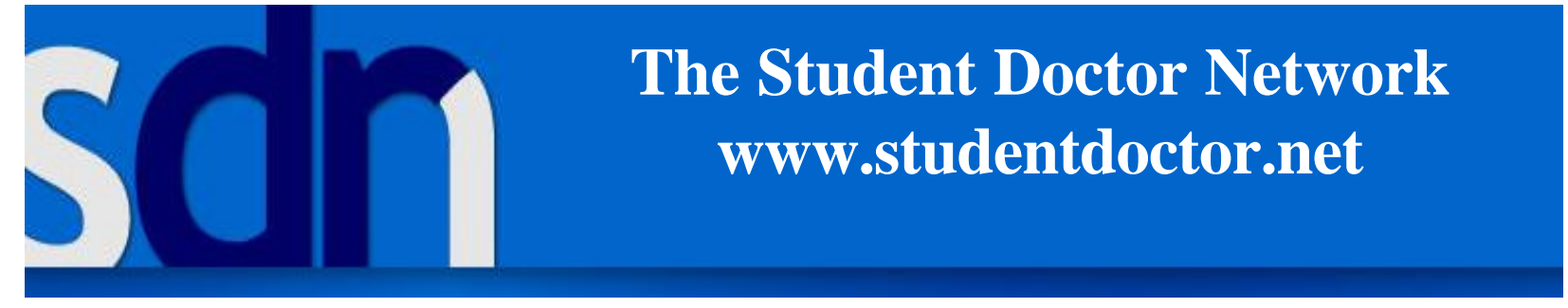

Come visit the MJM-co-hosted Student Research and Publishing Forum online at www.studentdoctor.net

For issues 9.1 and 9.2, the Student Doctor Network is proudly sponsoring a $\$ 500$ award for best MJM submission relating to biomedical education

\section{Contribute to the MJ M!}

